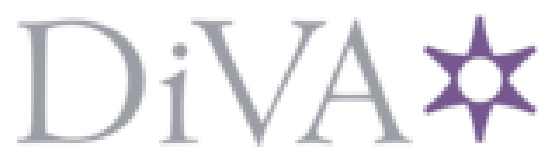

http://www.diva-portal.org

\title{
Postprint
}

This is the accepted version of a paper published in Dento-Maxillo-Facial Radiology. This paper has been peer-reviewed but does not include the final publisher proof-corrections or journal pagination.

Citation for the original published paper (version of record):

Ohman, A., Kivijärvi, K., Blombäck, U., Flygare, L. (2006)

Pre-operative radiographic evaluation of lower third molars with computed tomography..

Dento-Maxillo-Facial Radiology, 35(1): 30-5

http://dx.doi.org/10.1259/dmfr/58068337

Access to the published version may require subscription.

N.B. When citing this work, cite the original published paper.

Permanent link to this version:

http://urn.kb.se/resolve?urn=urn:nbn:se:umu:diva-89544 


\title{
RESEARCH
}

\section{Pre-operative radiographic evaluation of lower third molars with computed tomography}

\author{
A Öhman*,1, K Kivijärvi², U Blombäck ${ }^{2}$ and L Flygare ${ }^{1}$ \\ ${ }^{1}$ Department of Radiology, Sunderby Hospital, SE-971 80, Luleå, Sweden; ${ }^{2}$ Department of Maxillofacial Surgery, Sunderby Hospital, \\ SE-971 80 Luleå, Sweden
}

\begin{abstract}
Objectives: To report radiographic findings of examinations with computed tomography (CT) of impacted lower third molars with an intimate relation to the mandibular canal and to investigate how findings of a dark band across the roots of the lower third molar on panoramic views correspond to the CT findings.

Methods: CT images of 90 lower third molars (64 patients, mean age 34.6 years) in which plain radiography was insufficient to allow determination of the precise anatomical relations were reviewed retrospectively. Panoramic views of 88 teeth existed; these images were interpreted independent of the CT examinations with respect to presence of a dark band across the roots.

Results: In $31 \%$ of the CT images, the course of the mandibular canal was buccal, in $33 \%$ lingual, in $26 \%$ inferior, and in $10 \%$ inter-radicular. The tooth was in contact with the mandibular canal in $94 \%$ and with the lingual cortex in $86 \%$. In $23 \%$, grooving of the root by the canal was judged to be present. In $63 \%$ of the cases where a dark band across the roots was observed on panoramic radiographs, CT revealed grooving of the root.

Conclusions: Pre-operative CT of lower third molars is motivated in selected cases when plain radiography is inconclusive. The finding of a dark band across the roots on panoramic radiographs is an indicator of grooving of the tooth by the canal and justifies a pre-operative CT examination. The absence of dark bands on panoramic views does not exclude grooving of the roots.
\end{abstract}

Dentomaxillofacial Radiology (2006) 35, 30-35. doi: 10.1259/dmfr/58068337

Keywords: computed tomography, inferior alveolar nerve, radiography, third molar

\section{Introduction}

Removal of the lower third molar is one of the most common operations in oral surgical practice. In most cases it is a fairly straightforward procedure with few complications. However, when there is a close relationship between the tooth and the inferior alveolar nerve, damage to the nerve and dysaesthesia may occur. Many authors have reported the incidence of post-operative dysaesthesia of the inferior alveolar nerve after extraction of third molars. Temporary injuries in $0.4-5.5 \%$ and permanent nerve damage in $0.1-1.0 \%$ of cases of third molar extraction have been reported. ${ }^{1-4}$ However, these figures report the incidence of nerve damage when third molars of all degrees of difficulty are removed. When there is an intimate relationship between the tooth and the inferior alveolar nerve, the incidence of nerve injuries has been

*Correspondence to: Anders Öhman, Department of Radiology, Sunderby Hospital, SE-971 80 Luleå, Sweden; E-mail: anders.ohman@nll.se

Received 29 October 2004; revised 12 April 2005; accepted 12 May 2005 reported to be as high as $23-35 \% .^{5-7}$ Although permanent nerve injuries are infrequent, they are a serious discomfort for the patient when they occur. An accurate pre-operative radiographic examination is therefore considered indispensable before extraction of third molars. Ideally, this examination should help the surgeon to evaluate the difficulty of the operation and to choose the most appropriate surgical technique, for example, where to remove bone, how to split the tooth, and in what direction the roots can be lifted. In most cases, a conventional radiographic examination with plain radiography will suffice. In the most complicated cases, however, conventional radiography has its shortcomings, and relevant anatomical relationships cannot be accurately displayed. The need for more advanced techniques that can display anatomical relationships in three dimensions has thus emerged.

At our department, we began to use computed tomography (CT) frequently in 2001. The diagnostic 
potential of CT in these complicated cases has not been fully explored, and only a few papers cover this issue. ${ }^{8,9}$ In earlier studies based on plain radiography, several radiographic signs were proposed as predictors of nerve injuries. Rood and Shehab identified three signs as being significantly related to nerve injury: a dark band across the roots, a diversion of the canal, and an interruption of the white lines. ${ }^{6}$ A recent study has also confirmed these findings. ${ }^{10}$ A dark band across the roots is the sign that most often has been associated with grooving of the roots, and it has also consistently been associated with a high incidence of nerve injuries in several earlier studies. ${ }^{5-7,10,11}$

The primary aim of this study was to assess the relationship between the mandibular canal and the lower third molar radiographically in a selected group of patients with a suspected close relationship between the mandibular canal and the tooth. The second aim was to investigate whether the panoramic finding of a dark band across the roots correlated to findings of grooving of the roots on $\mathrm{CT}$.

\section{Materials and methods}

\section{Selection}

All patients who had undergone a CT examination of the mandibular region between January 2001 and August 2003 at the Department of Radiology, Sunderby Sjukhus, Luleå, Sweden, were retrospectively reviewed. Patients who had been referred for determination of the "position and anatomy of the lower third molar" were selected. Of this material, all patients who had other pathological conditions in the region of the lower third molar that were the main reason for requesting a $\mathrm{CT}$ examination were excluded. Thus, 16 cases of suspected cystic lesions and 1 case of a root that had been dislocated lingually at surgery prior to examination were

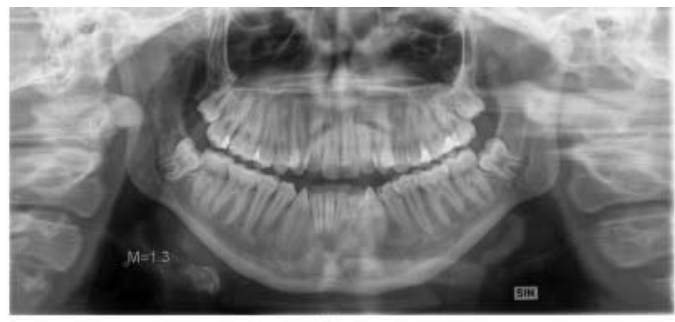

a

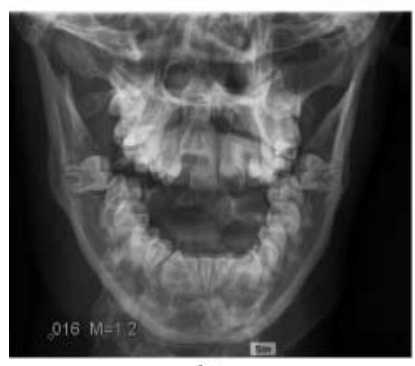

b

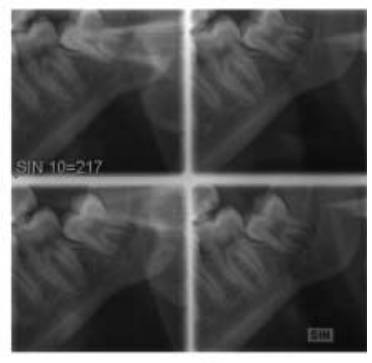

C
Figure 1 Conventional radiographic examination. (a) Panoramic (b) posterior-anterior (c) stereographic (scanograms)

excluded. One patient whose CT images were of poor quality due to metal artefacts was also excluded.

Sixty-four patients with a total of 90 lower third molars were thus selected. The group comprised 29 males and 35 females with a mean age of 34.6 years (range 17-65 years). Of the 90 teeth, 45 were left third molars and 45 right third molars.

We were able to obtain existing panoramic views of 88 of the 90 teeth. These panoramic views were interpreted independent of the CT examinations with respect to presence of a dark band across the roots.

\section{Imaging}

A panoramic view, a posterior-anterior view (PA), and stereographic views (Scanograms) were taken using the Scanora radiographic system (Soredex; Orion Corporation Ltd, Helsinki, Finland) (Figure 1). For the CT images, either Siemens Somatom Plus 4 Volume Zoom or Siemens Somatom Plus 4 (Siemens Medical Systems, Erlangen, Germany) was used. Our standard examination was made with the Somatom Plus 4 Volume Zoom in the axial plane, parallel to the occlusal plane (55 teeth). Exposure was made at $120 \mathrm{kV}, 100 \mathrm{mAs}$, with a collimation of $4 \mathrm{~mm} \times 1 \mathrm{~mm}$ and a pitch of 1 . From the raw data, $1 \mathrm{~mm}$ axial slices with an overlapping increment were reconstructed, and axial and coronal slices with a slice width of $2 \mathrm{~mm}$ were reformatted (Figure 2). The Somatom Plus 4 was used in all examinations prior to June 2002 (36 teeth). Direct coronal images were exposed at $120 \mathrm{kV}$ and $165 \mathrm{mAs}(110 \mathrm{~mA}$ and $1500 \mathrm{~ms})$ with a slice thickness of $2 \mathrm{~mm}$ (Figures 3 and $4 \mathrm{a}$ ).

In all cases all cuts of the third molar region were viewed. Rating parameters were set between the two radiologists prior to viewing the images. All cases were viewed by one radiologist (AÖ). Those cases that were

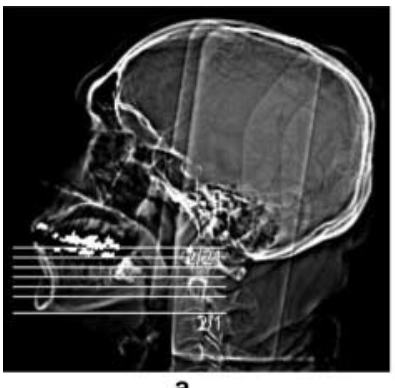

a

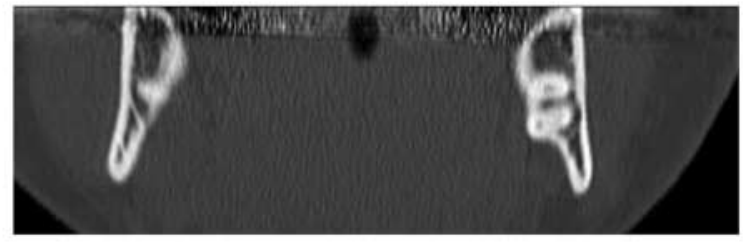

Figure 2 Example of a standard CT examination. (a) Examination performed in the axial plane parallel to the occlusal plane. (b) Axial. (c) Reformatted coronal image. The axial and coronal images show the inferior root penetrating the lingual cortex 
difficult to rate were reviewed together with a second radiologist (LF) and a consensus was always reached.

\section{Parameters}

The course of the mandibular canal: The course of the mandibular canal was classified as buccal, lingual, inferior, or inter-radicular. Buccal denoted a canal situated lateral to the lateral root surface of the tooth, lingual a canal situated medial to the medial root surface of the tooth, inferior a canal situated totally inferior to the roots, and interradicular a canal that in some part of its course passed between the roots. Cases where the mandibular canal had more than one course were classified according to the most difficult course taken by the canal in its passage of the tooth.

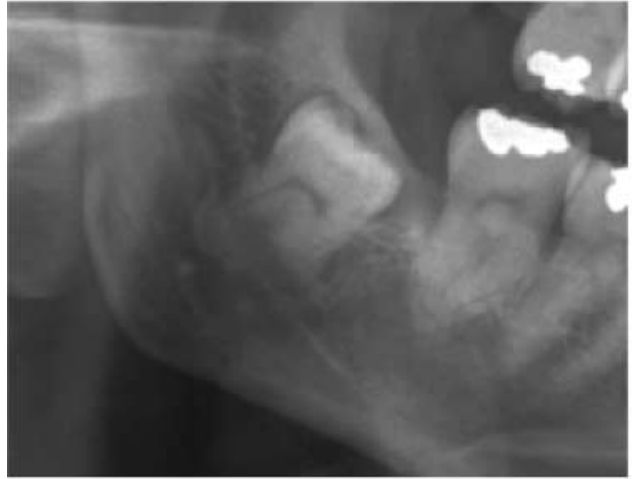

a

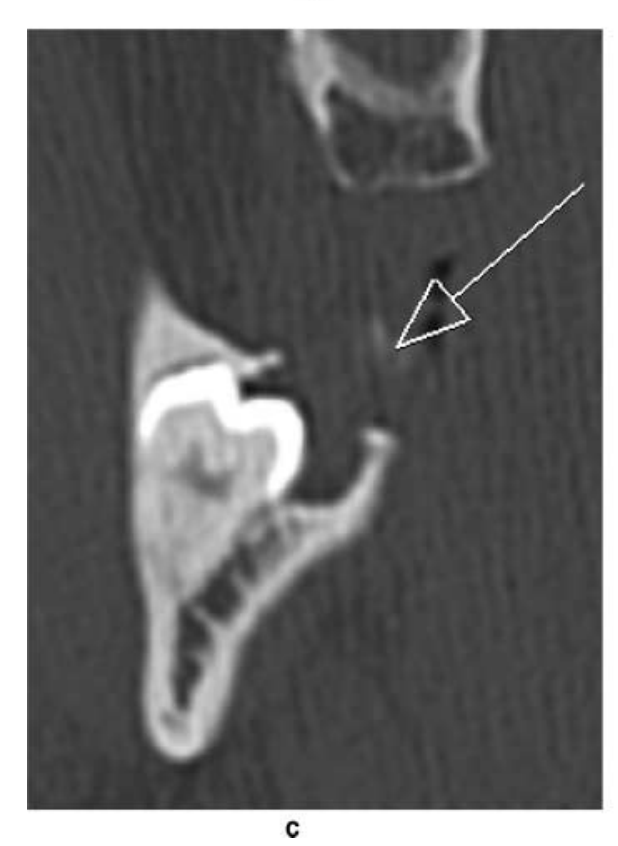

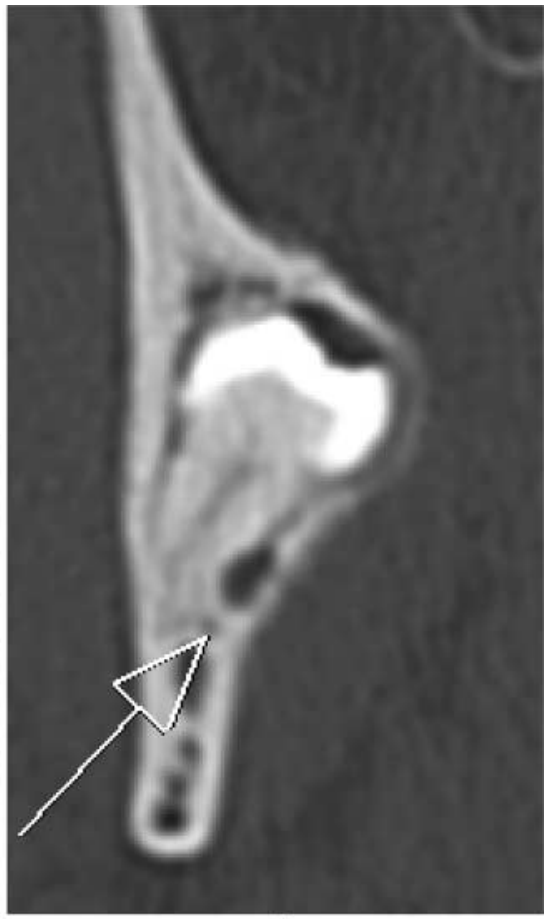

b

Figure 3 The relationship between a dark band across the roots and grooving of the roots. (a) Panoramic view (cropped) depicting a distinct dark band across the roots. (b) Direct coronal CT image depicting grooving of the root by the mandibular canal (arrow) on the lingual side. (c) Communication with the oral cavity is present (arrow) 


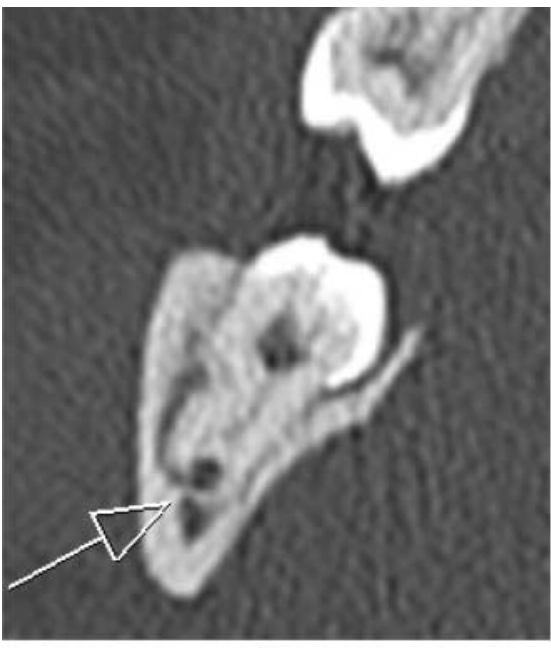

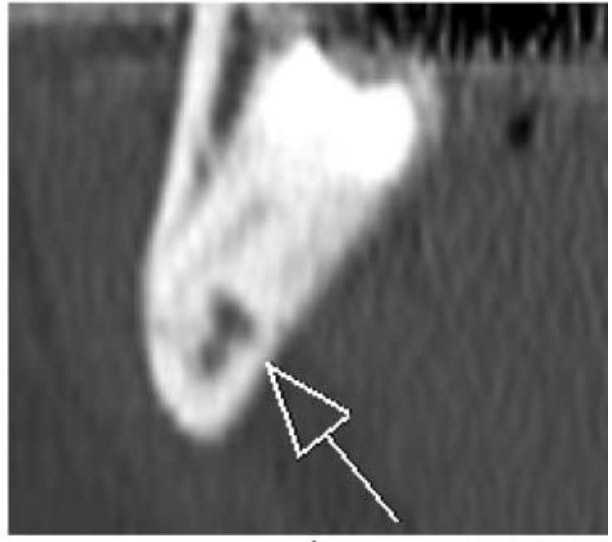

b

Figure 4 (a) Direct coronal image depicting an inter-radicular course and grooving of the roots by the mandibular canal (arrow). (b) Another patient where the distal root of 48 hooks around the mandibular canal (arrow)

Proximity of mandibular canal and lower third molar The proximity of the mandibular canal to the tooth was rated as "contact" or "no contact", contact meaning that no interstitial space or bone marrow existed between the tooth and an uncorticated or corticated mandibular canal. All other cases fell into the category "no contact".

Grooving and hook: "Grooving" indicated the presence of concavity or invagination of the root in which the canal was situated (Figures 3 and 4a). "Hook" indicated the presence of a deviation of the root apex, completely or partially encircling the mandibular canal (Figure 4b).

Proximity and effect on the lingual cortex: The relation of the tooth to the lingual cortex was scored as "contact" or "no contact".

Contact meant that some part of the tooth-the crown or the root-was in contact with the lingual cortex. No contact meant that no part of the tooth, crown, or root was in contact with the lingual cortex. If contact existed, a further classification into merely "contact" with the lingual cortex or a "thinning" or "perforation" of the lingual cortex was made. Thinning was defined as when some part of the tooth, crown, or root caused a local decrease in the thickness of the lingual cortex. Perforation was defined as when some part of the tooth, crown, or root penetrated the lingual cortex (Figure 2).

\section{Panoramic findings}

Whether a dark band was present across the roots was judged on panoramic views. Because there were no panoramic views for two patients, the group consisted of 88 teeth. A dark band was defined as a band of distinctly increased radiolucency where the mandibular canal overlapped the root of the lower third molar.

\section{Diagnostic methods}

Sensitivity and specificity are the true positive fraction and the true negative fraction, respectively. The likelihood ratio, which combines information from sensitivity and specificity, gives an indication of how much the odds of disease change based on a positive or a negative result. The definition for the positive likelihood ratio $\left(\mathrm{LR}^{+}\right)$is: ${ }^{12}$

$$
\left.\mathrm{LR}^{+}=\text {Sensitivity/(1 - Specificity }\right)
$$

and for the negative likelihood ratio $\left(\mathrm{LR}^{-}\right)$it is:

$$
\mathrm{LR}^{-}=(1-\text { Sensitivity }) / \text { Specificity }
$$

\section{Results}

The course of the mandibular canal as seen on the CT images was buccal in 31\%, lingual in 33\%, inferior in $26 \%$ and inter-radicular in $10 \%$ (Table 1).

We found 20 cases with combinations of different courses of the mandibular canal. Thirteen cases had a combined buccal and inferior course and six cases a lingual and inferior course; they were classified as buccal and lingual, respectively. One case where the combined course was buccal and between the roots was classified as interradicular.

Most of the teeth (84 teeth, 94\%) had contact with the mandibular canal.

Twenty-one $(23 \%)$ of the teeth had grooving, i.e. a concavity or invagination of the root in which the canal was situated, 7 (8\%) had a hook that partially or completely encircled the canal. Seventy-seven (86\%) had contact with the lingual cortex (Table 2).

Table 1 Course of the mandibular canal

\begin{tabular}{llllcc}
\hline & \multicolumn{2}{l}{ Course } & & & \\
\cline { 2 - 5 } & Buccal & Lingual & Inferior & Inter-radicular & Total \\
\hline No. & 28 & 30 & 23 & 9 & 90 \\
$\%$ & 31 & 33 & 26 & 10 & 100 \\
\hline
\end{tabular}


In 16 of 88 teeth $(18 \%)$, the panoramic view revealed a dark band. In all cases with a dark band, contact between the canal and root were seen. Ten of the 16 teeth $(63 \%)$ with a dark band had grooving (Table 3 ). Table 4 shows the statistical relationship between the dark band on panoramic view and grooving of the root.

The majority of the teeth with grooving $(12 / 21,57 \%)$ were associated with a mandibular canal that was lingual to the tooth, while the number with a buccal course was almost equal to that with an inter-radicular course (Table 5).

\section{Discussion}

In the present study, some results were not in accordance with previously published findings. We believe that this is mainly due to our strict selection criteria. Only cases where no conclusive diagnosis for the condition had been made, despite a complete radiological examination with conventional radiographic methods, were chosen for CT examination. Our aim was to put together a group of the most difficult cases of lower third molars with respect to radiographic assessment, surgical difficulty, and risk for

Table 2 Type and site of contact in the 77 teeth that were observed to have contact with the lingual cortex

\begin{tabular}{lllll}
\hline & \multicolumn{4}{c}{ Tooth part in contact with lingual } \\
& Cortex & \\
\cline { 2 - 4 } Type of contact & Crown & Root & Crown + Root & Total \\
\hline Plain contact & 1 & 13 & 0 & 14 \\
Thinning & 4 & 44 & 1 & 49 \\
Penetration & 3 & 10 & 1 & 14 \\
Total & 8 & 67 & 2 & 77 \\
\hline
\end{tabular}

Table 3 Relationship between dark band and grooving

\begin{tabular}{llll}
\hline & \multicolumn{2}{c}{ Presence of dark band } \\
\cline { 2 - 4 } & Yes & No & Total \\
\hline Grooving & 10 & 11 & 21 \\
No grooving & 6 & 61 & 67 \\
Total & 16 & 72 & \\
\hline
\end{tabular}

Table 4 Dark band as predictor of grooving as derived from Table 3

\begin{tabular}{ll}
\hline Sensitivity & $48 \%$ \\
Specificity & $91 \%$ \\
Positive predictive value & $63 \%$ \\
Negative predictive value & $85 \%$ \\
Positive likelihood ratio & 5.32 \\
Negative likelihood ratio & 0.58
\end{tabular}

Table 5 Grooving and the course of the mandibular canal

\begin{tabular}{|c|c|c|c|c|}
\hline & \multicolumn{3}{|l|}{ Course } & \multirow[b]{2}{*}{ Total } \\
\hline & Buccal & Lingual & Inter-radicular & \\
\hline No. & 4 & 12 & 5 & 21 \\
\hline$\%$ & 19 & 57 & 24 & 100 \\
\hline
\end{tabular}

nerve damage. In a previous study on CT examinations of lower third molars, teeth were included based on the association with the mandibular canal as observed solely on panoramic radiographs. ${ }^{9}$ We used panoramic, PA, and stereographic views (Scanograms), and these views will, in most cases, suffice to determine the relationship between the tooth and the mandibular canal and thus exclude the necessity of a CT. We fully agree with the conclusions of Blaeser et al who stated that "the routine use of CT scans in cases of third molar extractions cannot be recommended". ${ }^{13}$

Our CT images were produced with two different units and consequently different imaging protocols. We found no clinically significant difference in imaging quality or diagnostic outcome between these two methods.

In all cases, the course of the mandibular canal could be established. We found that the tooth and the mandibular canal were in contact in $94 \%$ of the studied cases and in $100 \%$ of the cases where a dark band was present on panoramic views. This disagrees with Monaco et al who reported contact in $73 \%$ of the teeth that displayed "increased radiolucency" on panoramic view. ${ }^{10}$ However, they used only axial CT images, and in our experience, contact between the canal and the tooth are more easily judged on coronal images. We found a correlation between a dark band across the roots of the third molar on panoramic images and grooving of the tooth by the mandibular canal. In a consecutive series of patients referred for removal of lower third molars, grooving of the root was found in $12 \%$ of the cases. ${ }^{7}$ In our selected material, the ratio of grooving was twice as high (23\%) and when a dark band was present on panoramic views, we found grooving in $63 \%$ of the cases. That a dark band on a panoramic view was a predictor of grooving of the root by the canal had a positive likelihood ratio of 5.32 and a negative likelihood ratio of 0.58 as seen in Table 4 . Thus, absence of a dark band on panoramic views does not exclude grooving, but the occurrence of a dark band makes a CT examination, in our opinion, advisable if extraction of the tooth is planned.

It has been suggested that grooving of the tooth by the canal almost always occurs on the lingual side. ${ }^{5}$ Our material does not support this. Although we found a lingual course in the majority of the cases, a course between the roots or bucally was also fairly common. When grooving is present, the course of the canal must also be known to give the clinician an opportunity to adjust the surgical approach and thus minimize the risk of complications.

Nearly all previous studies on the course of the mandibular canal have reported a predominantly buccal course, typically at least $50 \% .^{9,14,15}$ In our material only $31 \%$ of the cases had a buccal course, and a lingual course was the most common. An inter-radicular course is difficult to determine with conventional methods, but Maegawa et al, who used CT in their study, found an inter-radicular course in only $4 \%$ of their cases. Another study that used stereographic views (Scanograms) found an inter-radicular course in $3 \%$ of the cases. ${ }^{15}$ We found an inter-radicular course in $10 \%$ of our cases, probably due to our strict selection criteria. 
We found that $88 \%$ of the teeth had contact with the lingual cortex, 54\% a thinning of the lingual cortex, and $16 \%$ perforation of the lingual cortex. This information is highly relevant for the surgeon and might serve to lower the risk of damage to the lingual nerve, fracturing of the lingual cortex, and dislocations of bone or tooth fragments to the soft tissues of the floor of mouth.

CT is able to depict the desired detailed knowledge of the anatomical relationships, and it is not unreasonable to believe that this radiographic method might reduce the incidence of nerve injuries. Whether this really is the case was not examined in this work, but we have observed a reduction in the incidence of persistent nerve dysaesthesia at our department since we introduced CT. During the years 1996-2000, before we began to use CT, a total of five cases of persistent nerve dysaesthesia after surgical removal of lower third molars occurred at the Department of Maxillofacial Surgery, Sunderby Hospital. Since the introduction of CT at the beginning of 2001, we have not had a single case of persistent dysaesthesia. To show a lower incidence of nerve damage when CT is used to evaluate lower third molars is a decisive step in the validation of the method, and further research in this field is needed.

Surgeons report that the more precise information available in the CT images has simplified the surgical procedure and made it considerably safer. Another benefit is that the patient can be given more adequate information about the procedure and the associated risk. Treatment should always be in collaboration with the patient. With the extra support that CT gives, the patient's subjective

\section{References}

1. Valmaseda-Castellon E, Berini-Aytes L, Gay-Escoda C. Inferior alveolar nerve damage after lower third molar surgical extraction: a prospective study of 1117 surgical extractions. Oral Surg Oral Med Oral Pathol Oral Radiol Endod 2001; 92: 377-383.

2. Carmichael FA, McGowan DA. Incidence of nerve damage following third molar removal: a West of Scotland Oral Surgery Research Group study. Br J Oral Maxillofac Surg 1992; 30: 78-82.

3. Smith AC, Barry SE, Chiong AY, Hadzakis D, Kha SL, Mok SC, et al. Inferior alveolar nerve damage following removal of mandibular third molar teeth. A prospective study using panoramic radiography. Aust Dent J 1997; 42: 149-152.

4. Swanson AE. Incidence of inferior alveolar nerve injury in mandibular third molar surgery. J Can Dent Assoc 1991; 57: 327-328.

5. Howe GL, Poyton HG. Prevention of damage to the inferior dental nerve during the extraction of mandibular third molars. Br Dent $J$ 1960; 199: 355-363.

6. Rood JP, Shehab BA. The radiological prediction of inferior alveolar nerve injury during third molar surgery. Br J Oral Maxillofac Surg 1990; 28: $20-25$.

7. Rud J. Third molar surgery: relationship of root to mandibular canal and injuries to the inferior dental nerve. Tandlaegebladet 1983; 87: 619-631.

8. Feifel H, Riediger D, Gustorf-Aeckerle R. High resolution computed tomography of the inferior alveolar and lingual nerves. Neuroradiology 1994; 36: 236-238. degree of problem in combination with the complexity of the anatomy can be decisive for whether or not the patient wishes to continue with the operation.

One obvious drawback with CT is the higher dose of radiation that the patient receives compared with conventional radiography. A detailed study of radiation doses was not a part of this study. However, the $\mathrm{CTDI}_{\mathrm{vol}}$ was approximately $28 \mathrm{mGy}$ and the dose-length product was between $138 \mathrm{mGy} \mathrm{cm}$ and $149 \mathrm{mGy} \mathrm{cm}$ as given by the manufacturers. These values correspond to an estimated effective dose of approximately $0.30 \mathrm{mSv}$.

Our aim has been to reduce the amount of radiation as much as possible by producing images whose quality is only sufficient to solve the diagnostic task. Compared with earlier studies, ${ }^{8,9}$ our protocol significantly reduces the dose to the patient. We found contact between the tooth and the mandibular canal in $94 \%$ of our cases, which we see as a sign that our selection criteria are strict. A strict selection of cases may possibly be the most important factor for reducing the population dose of radiation.

\section{Conclusion}

We find that pre-operative CT of lower third molars is motivated in selected cases where plain radiography is inconclusive. A finding of a dark band on plain radiographs is an indicator of grooving of the tooth by the canal and justifies pre-operative CT examination. Absence of a dark band on plain radiographs does not exclude grooving of the roots.
9. Maegawa H, Sano K, Kitagawa Y, Ogasawara T, Miyauchi K, Sekine $\mathrm{J}$, et al. Preoperative assessment of the relationship between the mandibular third molar and the mandibular canal by axial computed tomography with coronal and sagittal reconstruction. Oral Surg Oral Med Oral Pathol Oral Radiol Endod 2003; 96: 639-646.

10. Monaco G, Montevecchi M, Bonetti GA, Gatto MR, Checchi L. Reliability of panoramic radiography in evaluating the topographic relationship between the mandibular canal and impacted third molars. J Am Dent Assoc 2004; 135: 312-318.

11. Kipp DP, Goldstein BH, Weiss Jr WW. Dysesthesia after mandibular third molar surgery: a retrospective study and analysis of 1,377 surgical procedures. J Am Dent Assoc 1980; 100: 185-192.

12. Radack KL, Rouan G, Hedges J. The likelihood ratio. An improved measure for reporting and evaluating diagnostic test results. Arch Pathol Lab Med 1986; 110: 689-693.

13. Blaeser BF, August MA, Donoff RB, Kaban LB, Dodson TB. Panoramic radiographic risk factors for inferior alveolar nerve injury after third molar extraction. J Oral Maxillofac Surg 2003; 61: 417-421.

14. Kaeppler G. Conventional cross-sectional tomographic evaluation of mandibular third molars. Quintessence Int 2000; 31: 49-56.

15. Tammisalo T, Happonen RP, Tammisalo EH. Stereographic assessment of mandibular canal in relation to the roots of impacted lower third molar using multiprojection narrow beam radiography. Int $J$ Oral Maxillofac Surg 1992; 21: 85-89. 